\title{
Mechanical and electronic-structure properties of compressed CdSe tetrapod nanocrystals
}

\author{
Joshua Schrier* and Byounghak Lee and Lin-Wang Wang \\ Computational Research Division, Lawrence Berkeley National Laboratory, Berkeley, California 94720 USA
}

\begin{abstract}
The coupling of mechanical and optical properties in semiconductor nanostructures can potentially lead to new types of devices. This work describes our theoretical examination of the mechanical properties of CdSe tetrapods under directional forces, such as may be induced by AFM tips. In addition to studying the general behavior of the mechanical properties under modifications of geometry, nanocrystal-substrate interaction, and dimensional scaling, our calculations indicate that mechanical deformations do not lead to large changes in the band-edge state eigenenergies, and have only a weak effect on the oscillator strengths of the lowest energy transitions.
\end{abstract}

\section{INTRODUCTION}

Although there has been much experimental[1,2] and theoretical[3, 4] work on the effects of hydrostatic-pressure induced structural transformations in CdSe nanocrystals, it has only recently become possible to study the mechanical responses of nanocrystalline tetrapod structures to directional forces.[5] Applying force from one direction, instead of hydrostatic pressure, will induce much more interesting mechanical deformation in a nanosystem, and the relatively complex geometry of a tetrapod provides more degree of freedom and possibilities for the system to deform. These tetrapod-shaped nanocrystals are interesting not only for their basic properties,[6] but also because they have already been used in applications such as single-electron transistor devices[7] and organic/inorganic hybrid photovoltaics. [8, 9]

In this paper, we apply theoretical simulation to study the mechanical deformation of an CdSe tetrapod under compression. The general geometry, consists of three legs placed on a substrate with a compressive force applied to the vertical arm of the tetrapod, mimicking a recent experiment where the tip of an atomic-force microscope (AFM) was used to apply the force.[5] Through our theoretical calculations, we would like to understand how the mechanical properties of the nanostructure compare to macroscopic counterparts, and to examine the possibility for coupling

${ }^{*}$ Corresponding author:jschrier@lbl.gov 
between the mechanical and optical properties, which may be useful for device applications. More specifically, we address the following questions: (1) How does the interaction of the legs with the substrate affect the mechanical properties of the tetrapod? (2) Does the size of the core of the tetrapod have any significant effect on the tetrapod deformation? (3) Are there any scaling laws of the spring constant of the tetrapod with respect to the leg length and diameter? (4) How do the electron wavefunctions change under the deformation? (5) What changes in optical absorption occur due to mechanical deformation?

\section{METHODOLOGY}

\section{A. Mechanical simulation}

To construct the tetrapod, we have used the model where the core of the tetrapod consists of a zinc blende structure, and the legs consist of wurtzite structure materials, following Ref. 6, as opposed to the model of multiple wurtzite twinning on a sphalerite nucleus (e.g., Ref. 10). We consider only perfectly ordered structures, noting that recent experiments on ZnS nanocrystals indicate that structural disorder can lead to additional stiffening.[11]

To simulate the mechanical properties of the tetrapod, we used the valence force field (VFF) method containing nearest-neighbor bond-stretching, bond-angle bending and bond-length/bond-angle terms fitted to the experimental bulk elastic constants.[12, 13] This VFF model reproduces the elastic constants of the bulk CdSe, while it provides the atomic forces on each atom, and also includes the nonlinear elastic response terms. However, since this type of classical model cannot handle bond breaking, our calculations only concern the elastic regime.

To model the compression of the tetrapod, the top and bottom boundaries of the system were treated as impenetrable planes perpendicular to the vertical arm of the tetrapod, which were then incrementally pushed together, minimizing the VFF total energy with respect to all of the atom coordinates at each step. The numerical derivative of the energy (with respect to displacement), gives the force.

\section{B. Electronic structure}

To study the effects of strain on the electronic structure of tetrapods, we used the semi-empirical pseudopotential model (SEPM).[13] The effective electronic potential undergoes a substantial change when a strain is applied to the structure. The SEPM is fitted to yield the correct deformation potential of the bulk crystal. More specifically, the 
hydrostatic deformation potential for the energy gap is defined as

$$
a^{\alpha}=\frac{d E_{g}^{\alpha}}{d \ln v}
$$

where $\alpha$ indicates the interband transition (e.g., $\Gamma_{8 v} \rightarrow \Gamma_{6 c}, \Gamma_{8 v} \rightarrow L_{6 c}$, etc.), $E_{g}^{\alpha}$ is the transition energy, and $v$ is the volume of the unit cell. Another quantity that we use to fit the SEPM is the "absolute" volume deformation potential of the valence band maximum (VBM) defined as

$$
a^{V B M}=\frac{d E_{V B M}}{d \ln v} .
$$

While the absolute value of the VBM cannot be measured from experiment, it is quite well-known that the local density approximation (LDA) density functional calculation is accurate for valence band structure. Here, we use the LDA valence band deformation potential as one of the fitting criteria. Table I shows the hydrostatic deformation potential of zinc-blende CdSe. Having established the SEPM using deformation potentials, we calculated electron energies and wavefunctions of tetrapod structures under various strains using the folded spectrum method.[14] The resulting wavefunctions were then used for the calculation of the oscillator strengths.

\section{RESULTS AND DISCUSSION}

\section{A. Effects of fixed versus freely moving legs on the force constant}

Since the exact nature of the interaction between the nanocrystal, its surface ligands, and the substrate is a complicated and not well understood, as a first tentative step we examine here the two simplest possible consequences of different interactions, namely (a) very weak nanocrystal-substrate interactions - the horizontal leg are allowed to freely along the bottom plane; and (b) very strong nanocrystal-substrate interactions - the ends of the horizontal legs are fixed in place. In Figure 1 we compare the two cases for the specific example of a tetrapod comprised of four (wurtzite) legs each of $2.6 \mathrm{~nm}$ diameter and $21 \mathrm{~nm}$ long, attached to a $3.3 \mathrm{~nm}$ diameter (zinc-blende) core. In the case of the weak-interaction (free-legs), shown in Figure 1a, we see that the required force to compress the tetrapod has two distinct linear regimes. In the first regime, shown by the red line, and corresponding to a spring constant of 0.36 $\mathrm{N} / \mathrm{m}$ (determined from the slope of the red line), the displacement is accommodated by the legs sliding outward; the strain is localized primarily near the core and the portion of the horizontal legs nearest to the core, and to a lesser

extent in the vertical arm. At the second regime, shown as the blue line, the tetrapod has been completely flattened on to the surface; the additional displacement now leads only to compression of the vertical arm, and subsequently a 
much stiffer force constant of $10 \mathrm{~N} / \mathrm{m}$. In our simulation, the symmetry of the applied force directly perpendicular to the rod only causes a compression of the vertical arm, and does not result in buckling. In actual experiment, it is likely that the symmetry of the system will be broken, causing the vertical arm to buckle or tilt towards one direction. Next we examine the strong-interaction (fixed-legs) case, shown in Figure 1b. Unable to move freely, the horizontal legs undergo an S-shaped buckling. For the displacements up to $2.8 \mathrm{~nm}$, this would correspond to a spring constant of $4 \mathrm{~N} / \mathrm{m}$; beyond $3 \mathrm{~nm}$ displacement, the force is observed to be reduced. In reality, the buckling may proceed by fracture or plastic deformations which are not captured in our simulation. Displacements beyond $6 \mathrm{~nm}$, as in the previously described free-leg calculations above give rise to compression of the vertical arm, and thus the very steep increase in the force beyond this region, similar to that observed in the case of the free legs. The order of magnitude increase (as compared to the free case) of the spring constant in the initial regime indicates the importance of the substrate-nanocrystal interactions in determining the measured mechanical properties.

\section{B. Effects of core-size on the force constants}

As shown in Figure 1a, when the legs are allowed to move freely on the surface, the angles between the three bottom legs increases and the highest strain occurs near the central core of the tetrapod. This stimulated us to examine whether the size of the core (with a fixed arm diameter and length) might strongly affect the spring constant of the compression. As a specific example, we examined the cases of 3.3, 4.0, 4.7, and 5.4 nm diameter zinc-blende cores, joining four equal legs of $2.6 \mathrm{~nm}$ diameter by $21 \mathrm{~nm}$ length, allowing the horizontal legs to move freely. As in Figure 1a, the calculated forces were linear, and the least-squares linear-fit gave spring constants of $k=0.28$, $0.23,0.26$ and $0.23 \mathrm{~N} / \mathrm{m}$, respectively. We attribute the small variations to numerical rounding errors in the fitting procedure. Therefore, we conclude that increasing the diameter of the zinc-blende core (while keeping the diameter of the legs fixed) does not change the force behavior. From an experimental perspective, although TEM or AFM imaging of tetrapods may not resolve the exact size of the zinc-blende core, our results indicate that this should not be the cause of variability in the mechanical properties.

\section{Leg-dimension scaling of the force constants}

From the classical continuum mechanics of beams, the force constant scales as $k \propto r^{2} / L$, where $r$ is the leg radius and $L$ the length. Although the geometry of our sample is more complicated, a proportionality relation would allow 
us to scale our results up to much large experimental dimensions. To study this, we examined the cases of $2.6 \mathrm{~nm}$ diameter x 5.2, 10.5 and $21 \mathrm{~nm}$ long legs, as well as the case of 2.6, 3.3, and $4.0 \mathrm{~nm}$ diameter x $10.5 \mathrm{~nm}$ long leg structures, for both the free- and fixed- leg conditions described in Section III A. In Figure 2, we have plotted the force constant, obtained from a linear-fitting of the force in the first 5-20 $\AA$ of the compression calculation, against $r^{2}$ and $l^{-1}$. It is apparent that for both the free- and fixed- leg cases, the scaling the calculated spring constants for tetrapods is proportional to the radius-squared diameter of the core/legs, and roughly inversely proportional to the inverse of the rod length, although a linear fit for this latter underestimates the calculated spring constant at long leg lengths. Since the chemically synthesized tetrapods can have leg lengths as long as $150 \mathrm{~nm},[7]$ this scaling relationship will be useful to extrapolate for larger cases beyond the limits of computer simulated structures.

\section{Effects of mechanical deformation on the band edge wavefunction}

In this section, we will discuss the effects of mechanical deformation of the tetrapods on the band-edge wavefunctions, using as a specific example, the case of a tetrapod comprised of $2.6 \mathrm{~nm}$ diameter x $5.2 \mathrm{~nm}$ long legs, surrounding a 3.3 $\mathrm{nm}$ diameter core, consisting of $5055 \mathrm{Cd}$ and Se atoms and 1540 pseudo-hydrogen surface passivation atoms. Using the strain calculated from our VFF simulation, we obtain the electronic structure following the approach described in Section II B, allowing the horizontal legs of the tetrapod to move freely (as described in Section III A). In Figure 3 we show the strain and the wavefunctions of two extreme structures, one with no vertical pressure (Figure 3a-g) and the other in which the tetrapod has been flattened against the surface due to an applied force of $6.2 \mathrm{nN}$ (Figure $3 \mathrm{~h}-\mathrm{n}$ ).

As shown in the table of calculated eigenvalues, Table II, the optical band gap of the tetrapod is decreased under vertical stress, albeit by less than $40 \mathrm{meV}$. The main contribution to changes in the band gap results from valence band maximum, $\mathrm{VB}_{1}$. The conduction band minimum, $\mathrm{CB}_{1}$, wavefunction is localized at the central zinc-blende core (Figure 3(b)) and is hardly affected by the structural changes due to strain. When compressed (Figure 3i), the center of mass of $\mathrm{CB}_{1}$ moves slightly toward the bottom of the core, but the change in the energy is only at most $3 \mathrm{meV}$ even under the maximum pressure. The $\mathrm{VB}_{1}$ wavefunction (Figure 3e), on the other hand, has more weight outside of the central cell and is more sensitive to the strain in the leg region (as seen in comparison to Figure 31). Without pressure, the $\mathrm{VB}_{1}$ and $\mathrm{VB}_{2}$ states (Figs. 3e,f) are doubly degenerated. The pressure breaks the symmetry of the tetrapod structure and lifts the degeneracy (compare to Figs. 3l,m), resulting in the reduction of the band gap. 


\section{E. Oscillator strength}

Changes in electronic structure, in particular the lifting of degeneracy in VBM states, can be observed in optical absorption measurements. In Fig. 4, we shows the oscillator strength $\sum_{\alpha=x, y, z}\left|\left\langle i\left|p_{\alpha}\right| j\right\rangle\right|^{2}$ between states $\epsilon_{i}$ and $\epsilon_{j}$. The position of individual transition peak moves toward lower frequency with applied forces but the changes in relative position is much smaller. Overall, the transition probability decreases in amplitude with increasing force. When no force is applied, the left panels, the transitions $\mathrm{VB}_{1} \rightarrow \mathrm{CB}_{1}$ and $\mathrm{VB}_{2} \rightarrow \mathrm{CB}_{1}$ at $\Delta \epsilon=\epsilon_{i}-\epsilon_{j}=2.138 \mathrm{eV}$ are doubly degenerated. Upon applying force, the two peak are separated by as much as $3 \mathrm{meV}$ at $6.2 \mathrm{nN}$, the right panel of Fig. 4. More notable changes in absorption spectrum occur in transitions to upper conduction bands. As shown in Table II, $\mathrm{CB}_{2}$ and $\mathrm{CB}_{3}$ stay nearly degenerate even under large strain. Coupled with the degeneracy of $\mathrm{VB}_{1}$ and $\mathrm{VB}_{2}$, the peaks corresponding to $\mathrm{VB}_{1} \rightarrow \mathrm{CB}_{2,3}$ and $\mathrm{VB}_{2} \rightarrow \mathrm{CB}_{2,3}$ transition are clustered at $\Delta \epsilon \approx 2.294$. Strains separate these peaks by $5 \mathrm{meV}$ at $6.2 \mathrm{nN}$. Although small, this strain-induced splitting of transition peaks could be observed in single nanostructure optical spectroscopy experiments (e.g., Ref. 15).

\section{CONCLUSION}

In summary, we have examined some of the mechanical properties of CdSe tetrapod nanocrystals and the resulting effects on the electronic structure. We find that modifications of the core size do not substantially alter the measured force constants, but the dimensions of the legs and whether the legs are fixed upon the surface or allowed to move freely, can drastically alter the observed mechanical properties. Finally, we observe that these types of directional mechanical alter the hybridization of the band-edge wavefunction states, but do not lead to large changes in the band-edge state eigenenergies. This has only a weak - but measurable - effect on the oscillator strengths calculated for the lowest-energy transitions. These findings may prove useful for the design of devices based on these materials.

\section{Acknowledgments}

This work was supported by the U. S. Department of Energy under Contract No. DE-AC03-76SF00098, and used the resources of the National Energy Research Scientific Computing Center. We thank Drs. Peter Graf and Qingzhong Zhao (National Renewable Energy Laboratory) for providing the code to generate the uncompressed 
tetrapod geometries.

[1] C.-C. Chen, A. B. Herhold, C. S. Johnson, and A. P. Alivisatos, Science 276, 398 (1997).

[2] K. Jacobs, J. Wickham, and A. P. Alivisatos, J. Phys. Chem. B 106, 3759 (2002).

[3] M. Grünwald, E. Rabani, and C. Dellago, Phys. Rev. Lett. 96, 255701 (2006).

[4] N. J. Lee, R. K. Kalia, A. Nakano, and P. Vashishta, Appl. Phys. Lett. 89, 093101 (2006).

[5] M. Salmeron (2007), private communication.

[6] L. Manna, D. J. Milliron, A. Meisel, E. C. Scher, and A. P. Alivisatos, Nature Mater. 2, 382 (2003).

[7] Y. Cui, U. Banin, M. T. Björk, and A. P. Alivisatos, Nano Lett. 5, 1519 (2005).

[8] B. Sun, H. J. Snaith, A. S. Dhoot, S. Westenhoff, and N. C. Greenham, J. Appl. Phys. 97, 014914 (2005).

[9] I. Gur, N. A. Fromer, and A. P. Alivisatos, J. Phys. Chem. B 110, 25543 (2006).

[10] L. Carbone, S. Kudera, E. Carlino, W. J. Parak, C. Giannini, R. Cingolani, and L. Manna, JACS 128, 748 (2006).

[11] B. Gilbert, F. Huang, H. Zhang, G. A. Waychunas, and J. F. Banfield, Science 305, 651 (2004).

[12] C. Pryor, J. Kim, L. W. Wang, A. J. Williamson, and A. Zunger, J. Appl. Phys. 83, 2548 (1998).

[13] A. J. Williamson, L. W. Wang, and A. Zunger, Phys. Rev. B 62, 12963 (2000).

[14] L.-W. Wang and A. Zunger, J. Chem. Phys. 100, 2394 (1994).

[15] J. J. Peterson and T. D. Krauss, Nano Lett. 6, 510 (2006).

[16] O. Madelung, M. Schulz, and H. Weiss, eds., Intrinsic Properties of Group IV Elements and III-V, II-VI, and I-VII Compounds, vol. 22 Pt. a of Landolt-Bornstein, New Series (Springer, Berlin, 1987). 
Figures 


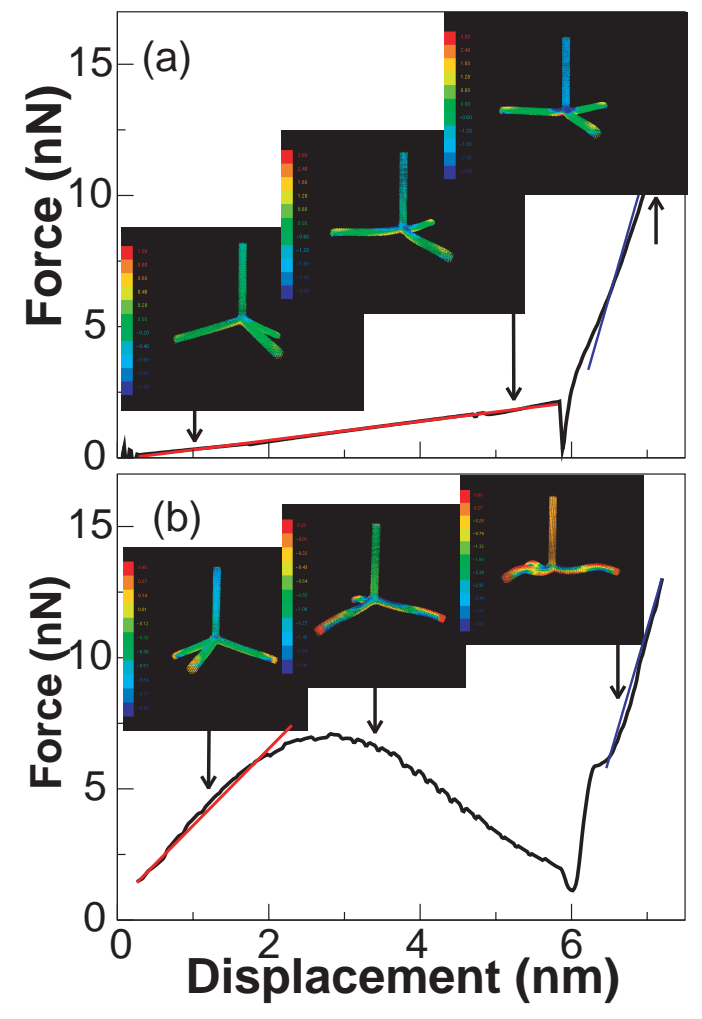

FIG. 1: (Color) Comparison of the effects of (a) weak nanocrystal-substrate interaction, modeled by freely-moving horizontal legs; and (b) strong nanocrystal-substrate interaction, modeled by fixed horizontal legs. Insets figures show the hydrostatic strain at each atom site. In the rightmost inset of (b), the color coding scale has been shifted to better illustrate the S-shaped buckling of the structure. 

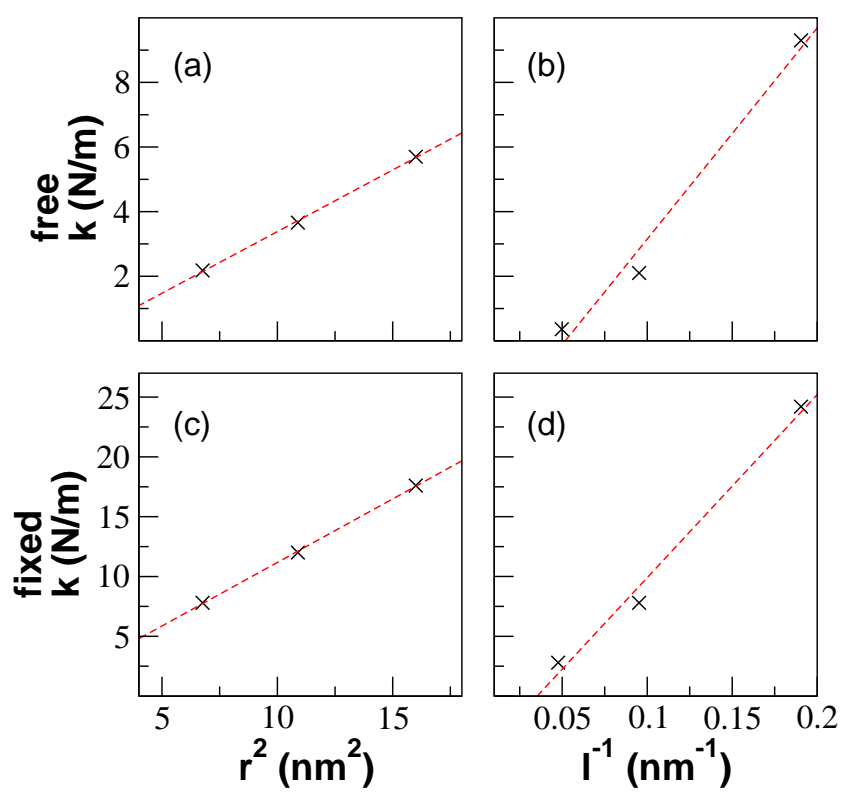

FIG. 2: Scaling of the force constant with respect to leg diameter and length, for both the (a,b) free- and (c,d) fixed- leg cases. (a) Free-leg force constant versus leg cross-sectional area ; (b) free-leg force constant versus leg length ;(c) fixed-leg force constant versus leg cross-sectional area ; (d) fixed-leg force constant versus leg length. 


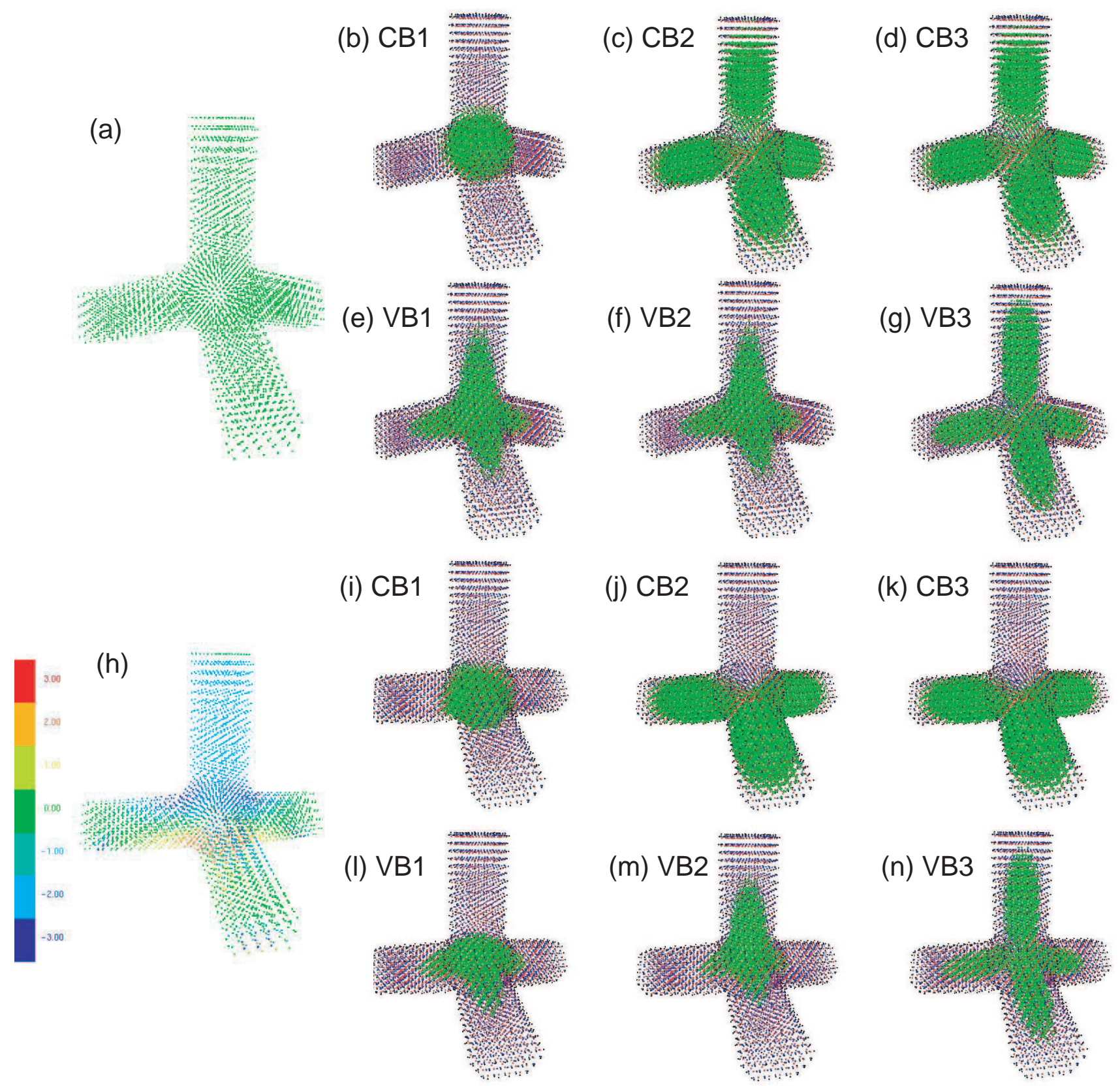

FIG. 3: (Color) Strain and band-edge wavefunction states for a tetrapod comprised of a 3.3 nm diameter core, surrounded by legs of $2.6 \mathrm{~nm}$ diameter by $5.2 \mathrm{~nm}$ long. Insets (a-g) show the results for the uncompressed (0.0 $\mathrm{nN}$ applied force) tetrapod. (a) Diagram of the tetrapod (which is completely unstrained); (b-d) Plot of the electron density for the first three conduction band (CB) edge states; (e-g) Plot of the hole density for the first three valence band (VB) edge states. Insets (h-n) show the results for a tetrapod completely flattened against the surface, resulting from an applied force of $6.2 \mathrm{nN}$, after allowing the horizontal legs to move freely (h) Hydrostatic strain at each atom site, represented as the percentage change in the local tetrahedron ; (i-k) CB states ; (l-n) VB states. 


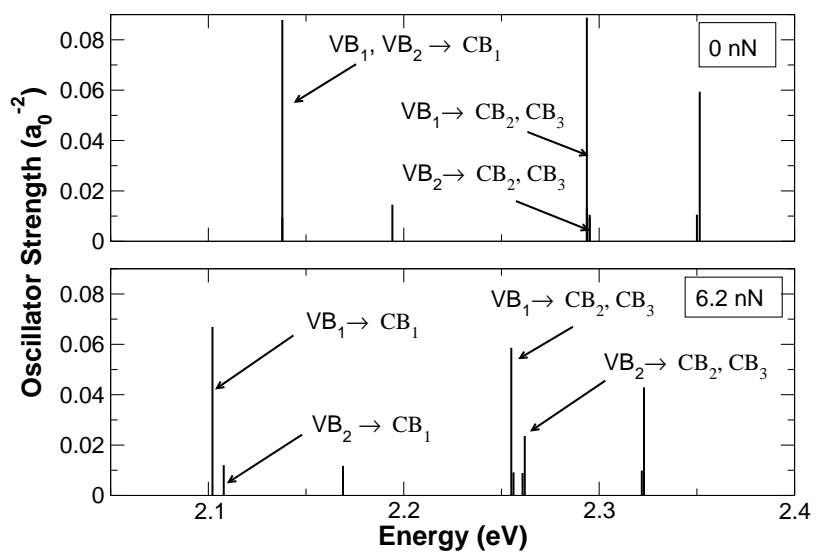

FIG. 4: Oscillator strength of a tetrapod comprised of a $3.3 \mathrm{~nm}$ diameter core, surrounded by legs of $2.6 \mathrm{~nm}$ diameter by $5.2 \mathrm{~nm}$ long. The top figure shows the results for an unstrained ( $0 \mathrm{nN}$ applied force) tetrapod (corresponding to strain and wavefunctions plotted in Figure 3a-g), and the bottom figure shows the results for a strained (6.2 $\mathrm{nN}$ applied force) tetrapod (corresponding to Figure 3h-n). 
Tables 
TABLE I: Deformation potential of zinc-blende CdSe. (eV)

\begin{tabular}{|c|c|c|c|}
\hline & SEPM & LDA & Expt. $^{a}$ \\
\hline \hline$a^{\Gamma_{{ } \rightarrow} \rightarrow \Gamma_{6 c}}$ & -3.07 & -1.96 & -3.07 \\
\hline$a^{V B M}$ & -1.87 & -1.81 & \\
\hline
\end{tabular}

${ }^{a}$ Reference 16. 
TABLE II: Eigenenergies of CdSe tetrapod in eV, calculated in Section IIID. $E_{g}$ is the energy gap between the conduction band minimum, $\mathrm{CB}_{1}$, and the valence band maximum, $\mathrm{VB}_{1}$.

\begin{tabular}{|c|c|c|c|c|c|c|c|}
\hline force $(\mathrm{nN})$ & $\mathrm{VB}_{3}$ & $\mathrm{VB}_{2}$ & $\mathrm{VB}_{1}$ & $\mathrm{CB}_{1}$ & $\mathrm{CB}_{2}$ & $\mathrm{CB}_{3}$ & $E_{g}$ \\
\hline \hline 0.0 & -4.742 & -4.688 & -4.688 & -2.550 & -2.394 & -2.393 & 2.137 \\
\hline 2.5 & -4.736 & -4.683 & -4.680 & -2.549 & -2.395 & -2.394 & 2.131 \\
\hline 6.2 & -4.722 & -4.660 & -4.655 & -2.553 & -2.400 & -2.399 & 2.102 \\
\hline
\end{tabular}

